\title{
Muskelerkrankungen diagnostizieren! Behandlung ist häufig möglich
}

$\mathrm{M}$ uskelerkrankungen umfassen heute eine nahezu unüberschaubare Vielzahl klinisch und ätiologisch unterschiedlicher Erkrankungen. Das Manifestationsalter reicht dabei von der Kindheit bis zum späten Erwachsenenalter. Neben den meist gut zu behandelnden erworbenen Myopathien gibt es viele genetisch bedingte, die noch immer allenfalls symptomatisch zu beeinflussen sind. Bei diesen nicht heilbaren Erkrankungen trifft man auch heute nicht selten auf die Meinung, dass eine exakte Diagnosestellung aufgrund fehlender therapeutischer Konsequenz nicht notwendig, allenfalls durch die Möglichkeit einer genetischen Beratung gerechtfertigt sei. Diese Ansicht verkennt, dass sich ärztliches Handeln keineswegs allein auf chirurgische oder medikamentöse Therapie beschränkt. Ebenso wichtig und hilfreich kann es sein, dem Patienten sagen zu können, dass sein Leiden keine unverstandene Behinderung und mystisch dunkle Bedrohung darstellt, sondern diagnostizierbar, benennbar und klassifizierbar ist. Allein das Verstehen der Erkrankung ermöglicht eine rational begründete Prognose die, auch wenn sie ungünstig ist, dem Patienten die Auseinandersetzung mit seinem Schicksal erlaubt.

Die molekular- und zellbiologischen Fortschritte haben in den vergangenen Jahrzehnten ein besseres und teilweise auch grundlegend neues Verständnis der Pathogenese vieler Myopathien ermöglicht. Dies hat dazu geführt, dass sowohl neue Krankheitsentitäten identifiziert werden konnten, als auch bekannte Erkrankungen neu klassifiziert werden mussten.

Das vorliegende Themenheft versammelt Beiträge von Mitarbeitern der Neurologischen Universitätsklinik
Halle/Saale: Stephan Neudecker gibt in seinem Beitrag eine Übersicht zur Klassifikation der verschiedenen Myopathien, wobei auch die große Vielfalt dieser Erkrankungen erkennbar wird. Malte Kornhuber stellt die Grundzüge des diagnostischen Vorgehens bei Myopathien dar.

In den Beiträgen von Kathrin Traufeller (entzündliche Myopathien), Katharina Eger (Störungen der neuromuskulären Übertragung) und Marcus Deschauer (mitochondriale Erkrankungen) werden ausgewählte Erkrankungsgruppen dargestellt, die aufgrund ihrer Häufigkeit und ihrer differenzialdiagnostischen Bedeutung auch in der nervenärztlichen Praxis von Interesse sind.

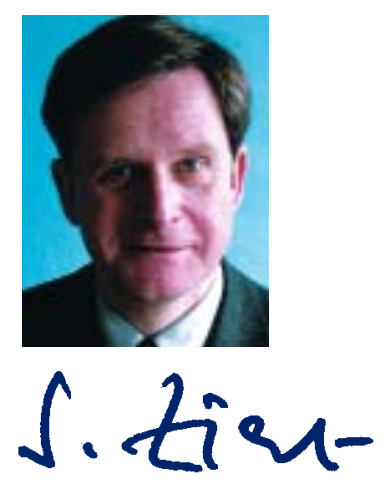

Prof. Dr. Stephan Zierz, Halle/Saale 